\title{
Osmotic sensitive characteristics of an LmpB mutant strain in cellular slime mould Dictyostelium discoideum
}

\author{
Tomoaki Abe, Maki Sukegawa \\ Department of Biological Engineering, Faculty of Science and Engineering, Ishinomaki Senshu University, \\ Ishinomaki 986-8580, Miyagi, Japan \\ Author for correspondence: T. Abe, tabe@isenshu-u.ac.jp
}

Summary: As one of the cellular slime mould mutants produced with restriction enzymes mediated integration (REMI), we isolated a strain that carries an insertion mutation in $\mathrm{LmpB}$ gene. $\mathrm{LmpB}$ is a lysosomal protein and suggested to be involved in the endosome maturation. Although some regulatory components associated with endosomes are commonly found in the contractile vacuoles, LmpB has not been suggested to be involved in the process of the osmotic regulation. However, when LmpB mutant cells were placed under osmotic stress, they developed morphologically aberrant large vacuoles in the cytoplasm and the viability of the cells was significantly reduced. The result implies that LmpB is not only involved in the endosome maturation, but also involved in the function of contractile vacuoles.

Key words: cellular slime moulds, osmotic regulation, endosomes, contractile vacuoles

\section{INTRODUCTION}

Cellular slime mould Dictyostelium discoideum has been known as a valuable model organism for studying a series of cell biological aspects, such as growth, motility, development, inter/intra-cellular signalling, intracellular structure, and vesicular transport. Upon starvation, the single cell amoebae transform into developmental stages that involve chemotaxis, cell sorting, cell differentiation, and formation of the multicellular body terminating at the culminant that consists of the spore head and the stalk tube. As their main food source, growing cells consume bacteria by phagocytosis. In addition, axenic mutant strains, which are frequently used in the laboratories, effectively take up liquid nutrients by macro-pinocytosis. Many kinds of proteins such as vacuolar $\mathrm{H}^{+}$-ATPase, myosins, LvsB, SNAP, NSF, PIP2, coronin, and Rab7 are known to be involved in these processes (Gotthardt 2002, Maniak 2003). LmpB is a member of the CD36/ LmpII protein superfamily and has a characteristic hair-pin structure, and C-terminus lysosomal targeting sequences. The intracellular co-localisation of $\mathrm{LmpB}$ with TRITC-dextran containing vesicles suggests that LmpB is involved in fusion or maturation steps of macropinosomes (Janssen et al. 2001). LmpB has also been reported to function in the phagocytotic event. At the early stage of phagosome maturation, nascent phagosomes are associated with coronin and rapidly receive LmpB (Gotthardt et al. 2002). Some of the regulatory components for endosome formation and lysosome fusion are commonly found in the process of contractile vacuole formation (Maniak 1999, Neuhaus et al. 2002). However, LmpB's association with contractile vacuole complexes has not been recognised.

In the stock of insertion mutant strains produced by REMI methods, we isolated an osmotic stress sensitive mutant, and identified the mutated gene for LmpB. The LmpB-mutant cells were able to grow normally in HL-5, the common liquid nutrient medium for $D$. discoideum. However, when cells were exposed to high osmotic shock, large sized-vacuoles were formed in the cytoplasm, and the survival rate of the cells was considerably reduced, compared to the wild-type cells.

\section{MATERIALS AND METHODS \\ Cell culture and cloning of mutant cells}

Wild-type Ax-2 cells and the mutant stock cells carrying hygromycin-resistant REMI-vectors were cultured in HL-5 (Watts and Ashworth, 1970) and in HL-5 containing $10 \mathrm{mg} / \mathrm{ml}$ hygromycin, respectively. The growing mutant stock cells at a density of apx. $2 \times 10^{6}$ cells/ml were collected and washed twice with KK2 buffer (16.5 mM KH $\mathrm{KO}_{4}, 3.8 \mathrm{mM} \mathrm{K} \mathrm{HPO}_{4}$, pH6.2). The washed cells were diluted with $K$. aerogenes suspension in KK2 buffer and plated on $25 \mathrm{~cm}$-diameter 
SM-agar plates (Sussman 1966) to obtain apx. 100 colonies/plate. The colonies were transferred into 24well culture plates (Tissue Culture Test Plates, No.92024, TPP, Switzland) in order to isolate individual clones. For phenotypical screening, duplicates of the original plates were prepared, and HL-5 medium in the individual wells was replaced to $250 \mathrm{mM}$ sucrose in order to expose the cells to high osmotic conditions. They were incubated at $22^{\circ} \mathrm{C}$ for 18 hours. Under these conditions, most of the wild-type cells were able to survive. The REMI-mutant clones that could not survive were selected, and the clones were isolated from the original culture plates.

\section{Identification of the mutated gene}

The mutated genes of selected clones were identified with inverted PCR (iPCR) (Keim, M. et al. 2004). The cells cultured in HL-5 were harvested for purification of genomic DNA with a DNeasy Tissue Kit (Qiagen, USA). The purified genomic DNA was digested with the restriction enzyme AluI, cleaned with a MinElute spin kit for cleaning (Qiagen, USA), and circularised with ligase (Promega, USA) incubating at $16{ }^{\circ} \mathrm{C}$ overnight. $1 \mu \mathrm{l}$ of circularised genomic DNA was used as the template for iPCR, and mixed with a PCR mixture containing Titanium Taq polymerase (ClonTech, Invitrogen, USA). After agarose-gel electrophoresis, the iPCR-amplified band was collected for DNA-extraction. $1 \mu$ of extracted DNA was used for cloning in pCR4 plasmid vectors with a TA-Cloning kit for sequencing (Invitrogen, USA). After bacterial transformation, the extracted plasmid vectors were applied into the DNA sequencer (ABI model 3100 , USA) for sequencing.

\section{Confirmation of REMI-mutation}

Following the results of the gene identification, a set of primers was synthesised and used for confirming the mutation in the target gene. The sequences of the primers used are as follows: LmpB415Rev: CCATCAG ATATAAGTCAAGCTCAAT, LmpB1009F: CTACAA ATGCTGCCATAATATATCC. Purified genomic DNA of the cells was used as the template for genomic PCR.

\section{Exposure of the cells to osmotic stress}

A $1 \mathrm{ml}$ suspension of cultured cells at the density of $3 \times 10^{6}$ cells $/ \mathrm{ml}$ was transferred to 24 -well culture plates (Tissue Culture Test Plates, No.92024, TPP, Switzland) and the medium was removed and replaced with 12.5$500 \mathrm{mM}$ sucrose solution. Cells were continuously cultured at $22{ }^{\circ} \mathrm{C}$ for 12 hours, and were observed with a phase-contrast inverted microscope (model Eclipse TS100, Nikon, Japan) equipped with a digital photographic camera system (model DS-L1, Nikon, Japan).

\section{RESULTS}

\section{Identification of LmpB gene in REMI mutant clones}

Through the screening process to find osmotic stress sensitive mutants, we isolated several candidate clones. In one of these candidates, we identified a LmpB mutant clone. With iPCR, the insertion point of REMI plasmid in LmpB gene was found at a DpnII site in the coding sequence (Fig.1 A). The sequence corresponds to the 374th amino acid in the total amino acids sequence length of 755 (Fig.1 B). For confirmation of the result, genomic PCR was performed using two primers inside of the coding regions. While the expected size of the single band of amplicons was visible with the genomic template of wild-type cells, no bands were detectable when the mutant genomic template was used, because the mutant amplicons were over $3 \mathrm{~kb}$ longer and were not effectively amplified (Fig.1 C).

\section{A.} ATGAAACATATTGGTAGAATTGTATCATTCCCAATTGGTTTAGTTTTAATCGCA GTTGGTATTATTATTTTTGTAGTTGTTAATCGTACAATTAAAGATGAATTTAAA AAAGCAGCCGTTGTAATTCCAGATAATGGTGCAGAAGAAATTGTTGATCCATGG GTTCGTTTCATTGGAAATGAAGGAGATCCAAACAATGTTAGAACCTATACCTTT ATGGCATATAATTTAACCAATCCAATTGAAACCATGCAAGGTCATTTACCAAAA TATCAAGAAGTTGGTCCATACAGTTACAATTACATCTATGAACGTATCAATGCA AACCTCTATGAAGATGATGAAAAATTAAGTTTCAAACTTTGGAAACGTTATTTC CCAATTGTTGCTGATGGTTATAGAGATCCAACCAAAGATACTATCTATCATTTC AATTTAGTATATGGTGCTGCCGTTAAACAAGCAGGTAGTGAAGTTGCACTTTCA GTAGCTTTAACAGCAGCTGCAATGGGTAAAATTATCACTGGTCTCACTGACCCA AGCTTTAAAGTTAAAGCTGGTTTCGCTGCTGCACCAACAGTTACTGCTGGAGCT TTTTCAAATCTTTTAACTGCAGCTGGTAATGACCCAGCTACTGCATGTGGACTT TGGCAAACTAGTACTAGTTCCAGTACTCCATTAGTTCCATTTTCTGTACCAATT ATTGCTGGTAGTCCATCAGATATAAGTCAAGCTCAATGTCAAGCATTATTTGAT CCATCAAATAAATTCTCTTTAACTGATCCAACAAATGTAGGTGTCTACCTTTTA AATCCAGCTGGATCCAAAGCTGCATTATTAGCTTCACCATTTGGTTTAACTAGT GTTCAAGCTGATTTAATTATGAAATATCAATTAGCATTAACATCAACTTTTGTA 
CCAACAACTCTTGTTTCTAGATTTGCTGAATGCTCTGATCCAAAAACTTGTACT AATAATCCACTTTACTTTGGTCTTTTACAATGGGCTAAAAATCCAAGTCTTCTT GGGCAATCAGTTTTCGCAATTCCAAATTCAGGTGTTCCAGCAGCACCAGAGTTT GGTATTTATACAAGTAGTGAACTCAGTTTAACAAAAGCAGGATCACTTTTCCTA AATACTTCAACTATTAATCTCATTACACCAACTAGTATTGGTACAATTTTAGTT TATGGACAAAAACTTAAAGCTGATCCAACATCAATT . . . .

\section{B.}

MKHIGRIVSFPIGLVLIAVGI I IFVVVNRTIKDEFKKAAVVIPDNGAEEIVDPW VRFIGNEGDPNNVRTYTFMAYNLTNPIETMQGHLPKYQEVGPYSYNYIYERINA NLYEDDEKLSFKLWKRYFPIVADGYRDPTKDTIYHFNLVYGAAVKOAGSEVALS VALTAAAMGKIITGLTDPSFKVKAGFAAAPTVTAGAFSNLLTAAGNDPATACGL WQTSTSSSTPLVPFSVPI IAGSPSDISQAQCQALFDPSNKFSLTDPTNVGVYLL NPAGSKAALLASPFGLTSVQADLIMKYQLALTSTFVPTTLVSRFAECSDPKTCT NNPLYFGLLQWAKNPSLLGQSVFAIPNSGVPAAPEFGIYTSSELSLTKAGSLFL NTSTINLITPTSIGTILVYGOKLKADPTSIPPALYAPFSSGEFDAIVKYTGYIM AAFVDGDI IKNOIFKDYOGGPIVKHTVHDFLFNSTDPLLKLMYPDTPSAWVSSP LDNIQDVKVANATLHTDEIYTGVGDIDLVSSPITFEGEEELNYNKKIKVSGSFA EOLPPSYLSKDPEAPVNVFTDEFARSLSFRKEVGGNFGGIPYYRYRINESNWEI NPDYFOTIPYLLNLTSIKSGAPAYLSRPRLKGIDVGYYYKAGITDLINDDEDLD VFADYEPRSGKAIHGRYSLQVNTYIOGSDGTNSTLYNKYSAFRSDVVHPMFWGV NI IAATOEOIDILTKAYKVDSFRYAITVILIVVGGFLSLISGGLFVLDKIIDL *

C.

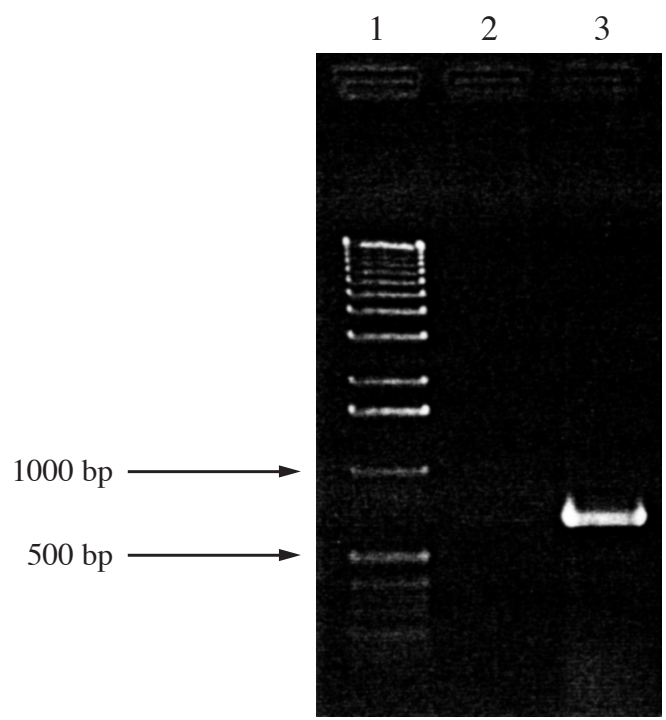

Figure 1. Insertion point of REMI vector in the mutant genome. (A) 5'-terminus portion of the coding sequence of LmpB (gene ID:DDB0187256). Reverse primer sequence used for genomic PCR confirming the insertion point is indicated with an arrow. Forward primer is further downstream of 594bp (refer to Materials and Methods). The insertion DpnII-sequence of REMI-vector is boxed. (B) The amino acid sequence of LmpB. Flanking amino acids of the insertion point at 374-375 a.a. are boxed. (C) Gel-photography of genomic PCR of LmpB coding sequence. Lane1 Marker, Lane2 LmpB mutant genomic template, Lane 3 wild-type genomic template.

\section{Sensitivity against high osmotic stress}

In order to test the effect of osmotic stress, mutant cells were incubated in 15-500 mM sucrose for 12 hours. During the incubation period, the cells were starved of necessary nutrients. Starved cellular slime mould cells are known to differentiate into aggregation competent cells. These cells are normally elongated and show polarized chemotactic movement towards cyclic AMP. Eventually, the cells first make multicellular forms called 'loose aggregates' (LA). We checked the survival rate of the cells, polarised shape of the cells, and the number of LA (Table 1). Typical changes in cell shape under osmotic stress are also shown in Fig. 2. The $D$. discoideum cells are osmotically neutral with 50-60 mM sucrose. Under the condition of $62.5 \mathrm{mM}$ sucrose, the shape of the mutant cells looked similar to the wild-type control cells (Fig. $2 \mathrm{C}$ and D). Their survival rate and the number of LA were not significantly different. When they were subjected to $15 \mathrm{mM}$ sucrose, the mutant cells' polarity and their ability to form aggregates seemed to be impaired (Fig. 2 A and B). Similar results were obtained with a high osmotic condition of 125-250 mM sucrose (Fig. $2 \mathrm{E}$ to $\mathrm{H}$ ), and particularly under the condition of $250 \mathrm{mM}$ sucrose (Fig. $2 \mathrm{G}$ and $\mathrm{H}$ ), a large number of mutant cells turned to be round, floated up from the substrate and eventually died. In the end, the survival rate was significantly reduced among the mutant cells. At $500 \mathrm{mM}$, both the wild-type and mutant cells could not survive. In $125 \mathrm{mM}$ sucurose, large sized vacuoles were visible in mutant cells (Fig.2 F).

\begin{tabular}{|c|c|c|c|c|}
\hline \multirow{2}{*}{$\begin{array}{c}\text { Cell } \\
\text { types }\end{array}$} & $\begin{array}{l}\text { sucrose } \\
\text { conc. } \\
(\mathrm{mM})\end{array}$ & $\begin{array}{l}\text { viability } \\
(\% \pm \mathrm{SD}, \mathrm{n}=3)\end{array}$ & $\begin{array}{l}\text { polarity } \\
(+ \text { :polar, } \\
\text {-:non-polar })\end{array}$ & $\begin{array}{l}\text { aggregates } \\
(+: \text { formed, } \\
- \text {-not formed })\end{array}$ \\
\hline \multirow{4}{*}{$\begin{array}{c}\text { Ax-2 } \\
\text { control }\end{array}$} & 15 & $95.2 \pm 2.4$ & + & + \\
\cline { 2 - 5 } & 62.5 & $98.1 \pm 3.2$ & + & + \\
\cline { 2 - 5 } & 125 & $94.9 \pm 3.6$ & + & + \\
\cline { 2 - 5 } & 250 & $43.7 \pm 3.6$ & + & + \\
\hline \multirow{4}{*}{$\begin{array}{c}\text { LmpB } \\
\text { mutant }\end{array}$} & 1500 & $3.5 \pm 2.3$ & - & - \\
\cline { 2 - 5 } & 62.5 & $74.5 \pm 6.4$ & - & + \\
\cline { 2 - 5 } & 125 & $75.5 \pm 2.8$ & - & - \\
\cline { 2 - 5 } & 250 & $20.4 \pm 3.3$ & - & N/A \\
\cline { 2 - 5 } & 500 & 0 & N/A & + \\
\hline
\end{tabular}

Table 1. Viability, morphological characteristics, and aggregate formation with LmpB mutant cells under osmotic stress. LmpB mutant cells and wild-type AX-2 control cells were harvested at the logarithmically growing stage in HL-5 medium. Cells were placed on 24-well culture plates and the medium was replaced with 15-500 $\mathrm{mM}$ sucrose. After 12 hours of incubation, the number of surviving cells was counted, and the morphology of the cells was monitored. 


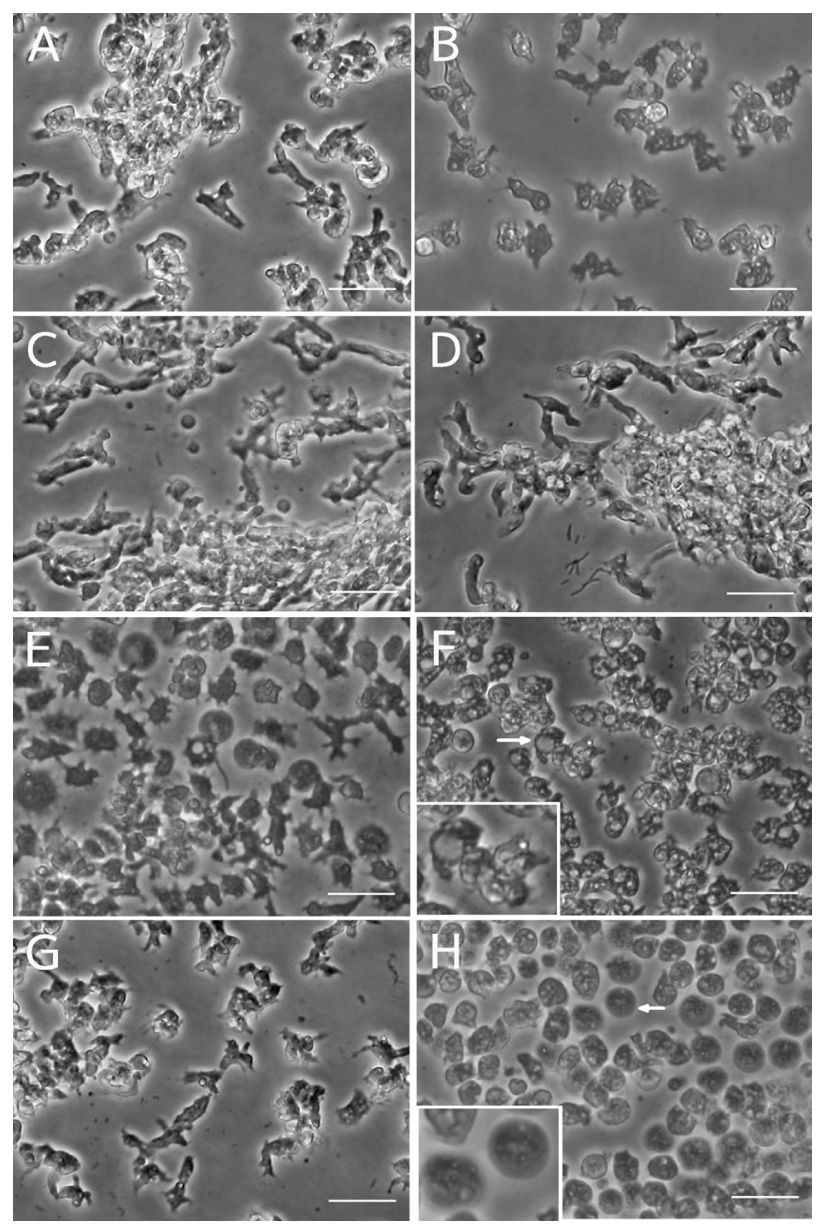

Figure 2. Morphology of LmpB mutant cells under the osmotic stress. LmpB mutant cells and wild-type Ax-2 cells were incubated with $15 \mathrm{mM}$ (A and B), $62.5 \mathrm{mM}$ (C and D), 125 $\mathrm{mM}(\mathrm{E}$ and $\mathrm{F})$ and $250 \mathrm{mM}(\mathrm{G}$ and $\mathrm{H})$ sucrose for 12 hours. A, C, E and G: wild-type Ax-2 cells, B, D, F and H: LmpB mutant cells. Inset pictures in $\mathrm{F}$ and $\mathrm{H}$ are the enlarged images of the cells indicated with the arrows. The bars indicate $25 \mu \mathrm{m}$.

\section{DISCUSSIONS}

We identified LmpB mutants and found that the mutant cells were highly sensitive to osmotic stress. LmpB has been reported as one of the lysosomal proteins and they are associated with endosomes (Janssen et al. 2001, Gotthardt et al. 2002). The possibilities that endosome associated proteins are also involved in the function of contractile vacuoles cannot be ruled out, as there are common regulatory proteins for both the processes (Maniak 1999, Neuhaus et al. 2002). RabD-like GTPase has been reported to be co-localised with vacuolar $\mathrm{H}^{+}-$ ATPase both in reticular membranes of the contractile vacuole $(\mathrm{CV})$ complex and in lysosomes (Bush et al. 1994, Harris and Cardelli 2002). RabD-like GTPases are also known to be detectable in early endocytic vesicles. For collecting water molecules through spongeomes, vacuolar H+-pumps are suggested to be important (Heuser et al, 1993). LmpB may be involved in providing functioning molecules like vacuolar $\mathrm{H}^{+}$-pumps to the $\mathrm{CVs}$ through lysosome fusion to CVs.

As shown in Fig. 2 F, large vacuoles were observed with mutant cells incubated in $125 \mathrm{mM}$ sucrose. We performed preliminary examination of pulse labelling of the vacuoles with FITC-dextran at various time points of incubation in $125 \mathrm{mM}$ sucrose. However, we could not detect the labelling. The result implies that these vacuoles may not be macro-pinosomes, but there remains a possibility that they may be aberrantly enlarged contractile vacuoles. For the controlling mechanisms of the function of $\mathrm{CVs}$, drainin and disgorgin have been reported to regulate several Rab-GTPases and they colocalise in $\mathrm{CV}$ membranes. With drainin or disgorgin depleted mutants, CVs are not able to fuse the plasma membrane, therefore they aberrantly enlarge (Du et al. 2008). In order to find the characteristics of the vacuoles observed in Fig. 2 F, we need further examinations to see whether such Rab-GTPase effrectors may co-localise with these vacuoles.

\section{ACKNOWLEDGEMENTS}

The authors would like to thank Dr. R. M. Halberstadt for his critical reading of the manuscript. This work was partly supported by Ishinomaki Senshu University Research Grant.

\section{REFERENCES}

Bush, J., Nolta, K., Rodriguez-Paris, J., Kaufmann, N., O’Halloran, T., Ruscetti, T., Temesvari, L., Steck, T., Cardelli, J. (1994) A Rab4-like GTPase in Dictyostelium discoideum colocalizes with $\mathrm{V}-\mathrm{H}+$ in reticular membranes of the contractile vacuole complex and in lysosomes. $J$. Cell Sci. 107: 2801-2812.

Du, F., Edwards, K., Shen, Z., Sun, B., De Lozanne, A., Briggs, S., Firtel, R.A. (2008) Regulation of contractile vacuole formation and activity in Dictyostelium. EMBO J. 27(15):2064-2076.

Gotthardt, D. Warnatz, H.J., Henschel, O., Bruckert, F., Schleicher, M., Soldati, T. (2002) High-resolution dissection of phagosome maturation reveals distinct 
membrane trafficking phases. Mol. Biol. Cell 13: 35083520 .

Harris, E., Cardelli, J. (2002) RabD, a Dictyostelium Rab14related GTPase, regulates phagocytosis and homotypic phagosome and lysosome fusion. J Cell Sci. 115(18):37033713.

Heuser, J., Zhu, Q., Clarke, M. (1993) Proton pumps populate the contractile vacuoles of Dictyostelium amoebae. J. Cell Biol. 121: 1311-1327.

Janssen, K-P., Rost, R, Eichinger, L., Schleicher, M. (2001) Characterization of CD36/LIMPII homologues in Dictyostelium. J. Bio. Chem. 276: 38899-38910.

Keim, M., Williams, R.S.B., Harwood, A.J. (2004) An inverse PCR technique to rapidly isolate the flanking DNA of Dictyostelium insertion mutants. Mol. Biotechnol. 26: 221224.

Maniak, M. (2003) Fusion and fission events in the endocytic pathway of Dictyostelium. Traffic 4:1-5.

Maniak, M. (1999) Endocytic transit in Dictyostelium discoideum. Protoplasma 210: 25-30.

Neuhaus, E.M., Almers, W., Soldati, T. (2002) Morphology and dynamics of the endocytic pathway in Dictyostelium discoideum. Mol. Biol. Cell 13:1390-1407.

Sussman, M. (1966) Biochemical and genetic methods in the study of cellular slime mold development. In Prescott D. [ed.], Methods in Cell Physiology, vol.2: pp. 397-410. Academic Press, New York.

Watts, D.J., Ashworth, J.M. (1970) Growth of myxamoebae of the cellular slime mould Dictysostelium discoideum in axenic culture. Biochem. J. 119: 171-174.

Received: 10 June 2009/ Accepted: 17 August 2009 